\title{
Legionellosis following water birth in a hot tub in a Canadian neonate
}

\author{
Michelle Barton MD MSc, Brianna McKelvie MD MSc, Aaron Campigotto MD, Tara Mullowney MD
}

Cite as: CMAJ 2017 October 23;189:E1311-3. doi: 10.1503/cmaj.170711

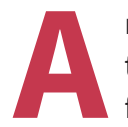

$\mathrm{n}$ eight-day-old full-term baby girl presented to our centre with a one-day history of fever, poor feeding and fussiness. There was no history of respiratory symptoms, drowsiness, seizures, vomiting or rash. Her prenatal history was unremarkable. Rupture of membranes had preceded vaginal delivery by three hours and there was no maternal fever. Under supervision of a midwife, the infant had been delivered underwater in a hot tub at home, which had been filled three days before delivery. Her birth weight was $3.49 \mathrm{~kg}$. She was vigorous at birth and breastfed well, with no concerns until the day of presentation.

Her temperature was $39.1^{\circ} \mathrm{C}$, with a heart rate of 172 beats/ $\mathrm{min}$, respiratory rate of 60 breaths $/ \mathrm{min}$ and an oxygen saturation of $97 \%$ on room air. Her physical examination was unremarkable except for some mildly increased work of breathing, and her chest radiograph showed right upper lobe pneumonia. We started ampicillin and cefotaxime after completing investigations for sepsis (Appendix 1, available at www.cmaj.ca/lookup/suppl/ doi:10.1503/cmaj.170711/-/DC1). Later that same day, we transferred our patient to the intensive care unit (ICU) with respiratory deterioration that required intubation. She had multiorgan fail-

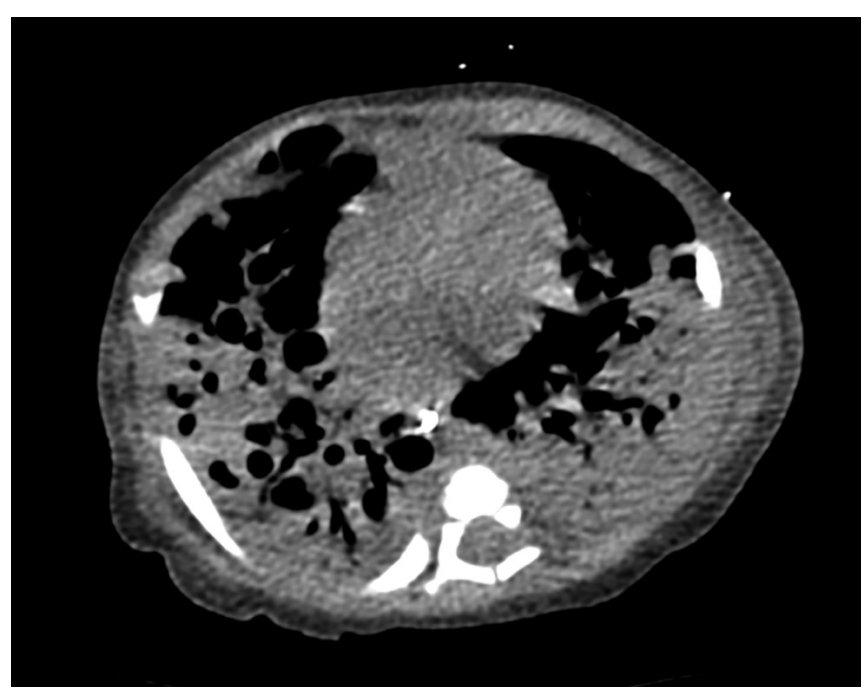

Figure 1: Computed tomography scan image showing extensive bilateral necrotizing pneumonia in an eight-day-old girl, after a water birth.

\section{KEY POINTS}

- Water immersion during labour and delivery may result in exposure to water-borne pathogens such as Legionella and Pseudomonas.

- Prefilling the tank days ahead of delivery, inadequately disinfecting birthing pools, using hot tubs and contaminated water sources, and heating water may increase the bacterial load of birthing water.

- Neonatal legionellosis presents as a potentially fatal sepsis syndrome characterized by severe pneumonia progressing to acute respiratory distress syndrome, septic shock, multiorgan failure and death in more than half of cases.

- Standard microbiologic investigations performed for neonatal sepsis will not identify Legionella species.

ure, acute respiratory distress syndrome, hematologic abnormalities and hemodynamic instability that required high doses of inotropic and pressor support.

While in the ICU, her antibiotics were changed to meropenem, vancomycin, azithromycin and acyclovir, with the latter discontinued once herpes simplex virus testing returned negative. Azithromycin was initially discontinued after seven days because of no apparent response. Appendix 1 summarizes our patient's course in the ICU. Cultures of blood and endotracheal aspirate taken before meropenem was started came back negative, although a Gram stain of our patient's endotracheal aspirate showed a marked inflammatory response in the absence of any organisms, which has been associated with Legionella infection. ${ }^{1}$ An erythematous macular rash developed on the patient's limbs on day 13 of her hospital stay and gradually became generalized over the next four days (Figure 1).

A computed tomography scan excluded congenital lesions but showed a necrotizing pneumonia (Figure 2). Respiratory samples were sent for routine culture and Legionella culture and a urine sample was sent for Legionella antigen. Once these cultures were sent, azithromycin was restarted on day 19 to cover Legionella. Respiratory cultures grew pan-sensitive Pseudomonas aeruginosa and meropenem-resistant, piperacillin-tazobactam-sensitive Cupriavidus gilardii. 


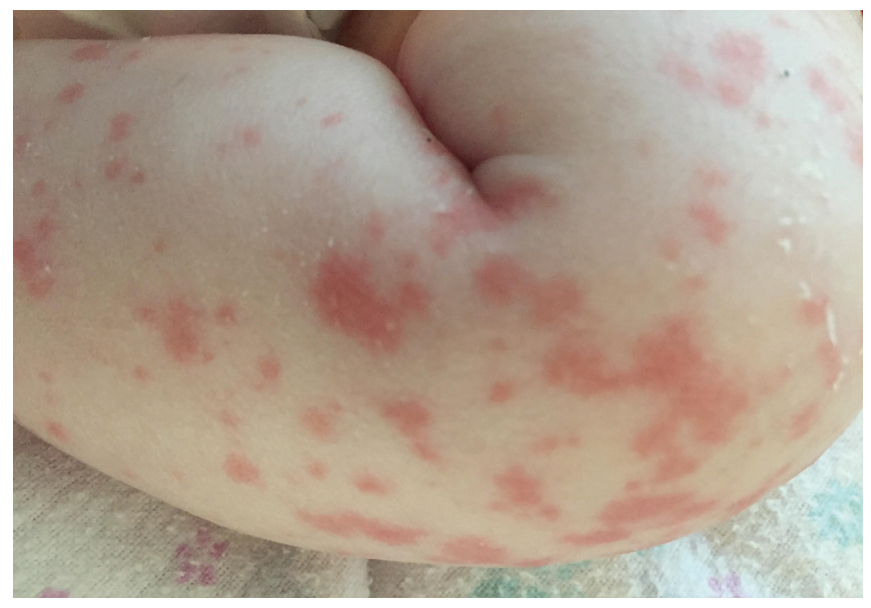

Figure 2: Photo of erythematous maculopapular rash on the left lower limb of an eight-day-old infant who had a water birth. The rash became generalized over four days.

Consequently, therapy was changed to piperacillin-tazobactam, and azithromycin was continued while we waited for the Legionella cultures, even though urine antigen testing for Legionella was negative. The cultures returned positive for Legionella pneumophila serotype 6 and we added rifampin for synergy. Improvements in ventilator settings were noted within 48 hours after azithromycin was started, and our patient was successfully extubated after having required mechanical ventilation for five weeks. At two months of age, she was discharged on home oxygen with close follow-up. Testing of the birthing water to confirm the source of Legionella infection was not possible, as the tub had been emptied and disinfected.

\section{Discussion}

Legionellosis is an uncommon diagnosis in the neonatal period. Cases have been reported sporadically, with up to 13 cases cited in a 2010 review $^{2}$ and as a part of a nosocomial outbreak in which nine neonates in a newborn unit were infected. ${ }^{3}$ Reports have documented legionellosis following water births., ${ }^{2,4-6}$ Although reports of neonatal legionellosis are few, it is possible that the disease is underrecognized and therefore underreported, given its similar presentation to more common causes of pneumonia, and the need for specialized, targeted microbiologic tests, which require the medical team to consider legionellosis on the differential diagnosis.

Legionella species are Gram-negative bacteria that proliferate in amebae living in fresh-water environments, especially at temperatures of $20^{\circ} \mathrm{C}$ to $42^{\circ} \mathrm{C}$, and are not killed unless the temperature exceeds $60^{\circ} \mathrm{C} .{ }^{1}$ Legionella pneumophila serogroup 1 represents the most common species and serogroup-causing disease. ${ }^{1,7}$ Infection begins 3 to 14 days after aspiration or inhalation of aerosolized droplets from contaminated water sources, especially in hosts with deficient immune systems. Legionella infections have the potential to cause severe lung injury through destruction of surfactant mediated by phospholipase A secretion. ${ }^{8}$ Disease is often multilobar and may become cavitary, as in our patient. ${ }^{2-4}$ Progression to respiratory failure is common $(67 \%$ among six outbreak cases with abnormal chest imaging ${ }^{3}$ ) and infants may end up requiring high-frequency oscillatory ventilation, inhaled nitric oxide or even extracorporeal life support. ${ }^{1}$ Endotoxemia may result in septic shock, coagulopathy and multiorgan failure. ${ }^{2,9}$ Nonspecific immune-mediated dermatological manifestations of legionellosis have been described in older patients, but are infrequent in neonates. ${ }^{9}$

Respiratory specimens that identify a marked inflammatory response on microscopy but fail to grow an organism on standard cultures can be an early clue. ${ }^{1}$ Isolation of Legionella species by culture is the gold standard, but requires the use of complex media - typically, buffered charcoal yeast extract medium supplemented with $0.1 \% \alpha$-ketoglutaric acid, as was performed in our case; ${ }^{9,10}$ nucleic acid-based methods are also often available for detection from respiratory tract specimens. ${ }^{11}$ Finally, urinary antigen testing may be employed for the diagnosis of Legionella pneumophila serogroup 1 infections; where a positive result has a specificity of $99 \%$ with minimal to no crossreactivity to non-Legionella pneumophila serogroup 1 serogroups; ${ }^{5,10,11}$ sensitivity can be as low as $70 \% .^{11}$

Treatment with fluoroquinolones or macrolides is recommended; the latter is the preferred therapy for neonatal disease. ${ }^{2,9}$ Therapy may be extended for 21 days, with rifampin added in severe cases. ${ }^{2,9}$ Overall mortality rates in neonates are up to $55 \%{ }^{12}$ and the disease may be uniformly fatal if untreated. ${ }^{2}$

The two other organisms isolated in this case, $P$. aeruginosa and Cupriavidus species, have been identified in water from birthing pools. ${ }^{5}$ Both are Gram-negative, glucosenonfermenting bacteria that can cause necrotizing pneumonia and bacteremia and are easily cultured from blood and respiratory specimens using standard cultures. ${ }^{13}$ The failure to culture these organisms from multiple blood cultures from our patient despite her septic shock suggested that the systemic features were likely not attributable to these organisms. Although Cupriavidus species are usually not pathogenic, opportunistic infections have been described; they show variable susceptibilities, and are often resistant to meropenem while sensitive to piperacillin-tazobactam. ${ }^{14,15}$

\section{Water births}

Several of the neonatal legionellosis reports have proven links to contaminated water systems, either at hospitals or at the infants' homes..$^{2-4}$ Infectious exposures have occurred through inhalation or aspiration of contaminated water in humidifiers, ${ }^{2,3}$ baths or birthing tanks ${ }^{2,4-6}$ used for water births.

Water births can reduce both the duration of the first stage of labour and analgesia requirements without evidence of harm to the mother. ${ }^{16}$ However, as data are insufficient to determine the risks and benefits during the second stage of labour and delivery, the American College of Obstetricians and Gynecologists has recommended that birth take place on land and not in water. ${ }^{16}$

Higher bacterial concentrations in the water increase the chance that even minute subclinical aspiration events could progress to severe pneumonia. Factors such as prefilling the tank days ahead of delivery, inadequately disinfecting the birthing tank, using a contaminated water source, using jetted tubs, and 
heating water all increase the bacterial load of the birthing water. ${ }^{17}$ Sources of bacteria in hot tubs include a contaminated domestic water source, debris falling into an uncovered tub, and biofilms within the piping system; the latter can pose challenges to decontamination.

Recommendations from the United Kingdom and the United States on water births now stress that jetted pools or pools with recirculating pumps should not be used and that tanks should be properly disinfected and, ideally, not be prefilled. ${ }^{17,18}$ However, there are safety concerns on the use of disinfectants, given the lack of data on the impact of potential aspiration or exposure of the neonate's skin and mucous membranes to chemicals used to disinfect tanks. Although adherence to infection-control strategies helps to limit risk, contamination may still occur from the water source..$^{19,20}$

Our case serves to highlight a severe and potentially fatal adverse neonatal outcome of underwater birth, especially when prefilled heated pools are used. Practitioners may wish to consider Legionella testing even if routine cultures identify a plausible pathogen in infants presenting with severe necrotizing pneumonia that progresses to respiratory failure within two weeks of an underwater birth.

\section{References}

1. Edelstein PH, Lück C. Chapter 49: Legionella. In Jorgensen JH, Pfaller MA, Carroll KC, et al., editors. Manual of Clinical Microbiology. 11th ed. Washington (DC): American Society of Microbiology; 2015:887-904. Available: www.asmscience. org/content/book/10.1128/9781555817381 (accessed 2017 June 2).

2. Shachor-Meyouhas $\mathrm{Y}$, Kassis I, Bamberger E, et al. Fatal hospital-acquired Legionella pneumonia in a neonate. Pediatr Infect Dis J 2010;29:280-1.

3. Yiallouros PK, Papadouri T, Karaoli C, et al. First outbreak of nosocomial Legionella infection in term neonates caused by a cold mist ultrasonic humidifier. Clin Infect Dis 2013;57:48-56.

4. Granseth G, Bhattarai R, Sylvester T, et al. Notes from the field: two cases of Legionnaires' disease in newborns after water births - Arizona, 2016. MMWR Morb Mortal Wkly Rep 2017;66:590-1.

5. Phin N, Cresswell T, Parry-Ford F; Incident Control Team. Case of Legionnaire's disease in a neonate following a home birth in a heated birthing pool, England, June 2014. Euro Surveill 2014;19:20857.

6. Fritschel E, Sanyal K, Threadgill H, et al. Fatal legionellosis after water birth. Texas, USA, 2014. Emerg Infect Dis 2015;21:130-2.

7. Flieger A, Gong S, Faigle M, et al. Novel phospholipase A activity secreted by Legionella species. J Bacteriol 2000;182:1321-7.

8. Moscatelli A, Buratti S, Castagnola E, et al. Severe neonatal Legionella pneumonia: full recovery after extracorporeal life support. Pediatrics 2015;136:e1043-6.

9. Rubin LG. Legionellosis. In Long SS, Pickering LL, Prober C, editors. Principles and practice of pediatric infectious diseases. 4th ed. London (UK): Churchill Livingston, Elsevier; 2012:912-5.

10. Fields BS, Benson RF, Besser RE. Legionella and Legionnaires' disease: 25 years of investigation. Clin Microbiol Rev 2002;15:506-26.

11. Mercante JW, Winchell JM. Current and emerging Legionella diagnostics for laboratory and outbreak investigations. Clin Microbiol Rev 2015;28:95-133.
12. Greenberg D, Chiou CC, Famigilleti R, et al. Problem pathogens: paediatric legionellosis - implications for improved diagnosis. Lancet Infect Dis 2006;6:529-35.

13. Yacoub AT, Halliday J, Katayama M, et al. Necrotizing pneumonia in cancer patients: a retrospective observational study, 1987-2011. Infect Dis Clin Pract 2015; 23:85-8.

14. Karafin M, Romagnoli M, Fink DL, et al. Fatal infection caused by Cupriavidus gilardii in a child with aplastic anemia. J Clin Microbiol 2010;48:1005-7.

15. Kobayashi T, Nakamura I, Fujita H, et al. First case report of infection due to Cupriavidus gilardii in a patient without immunodeficiency: a case report. BMC Infect Dis 2016;16:493.

16. American College of Obstetricians and Gynecologists' Committee on Obstetric Practice. Committee Opinion No. 679: Immersion in water during labor and delivery. Obstet Gynecol 2016;128:e231-6.

17. Collins SL, Afshar B, Walker JT, et al. Heated birthing pools as a source of Legionnairres disease. Epidemiol Infect 2016;144:796-802.

18. Fremgen $\mathrm{L}$. What we learned about waterbirth from a case of Legionella. Midwifery Today Int Midwife 2015;(115):33-4.

19. Teare L, Millership S. Legionella pneumophila serogroup 1 in a birthing pool. J Hosp Infect 2012;82:58-60.

20. Franzin L, Scolfaro C, Cabodi D, et al. Legionella pneumophila pneumonia in a newborn after water birth: a new mode of transmission. Clin Infect Dis 2001;33:e103-4.

\section{Competing interests: None declared.}

This article has been peer reviewed.

The authors have obtained consent from the parents of the patient.

Affiliations: Sections of Infectious Diseases (Barton), Paediatric Critical Care (McKelvie), and Respirology (Mullowney), Department of Paediatrics; Division of Microbiology (Campigotto), Department of Pathology and Laboratory Medicine, London Health Sciences Centre, Western University, London, Ont.

Contributors: Brianna McKelvie, Aaron Campigotto, Tara Mullowney and Michelle Barton contributed to the conception and design of the work. Michelle Barton and Brianna Mckelvie drafted the manuscript and all authors participated in critically editing and revising the document. All of the authors gave final approval of the version to be published and agree to be accountable for all aspects of the work.

Correspondence to: Michelle Barton, mabartonf@uwo.ca

The section Cases presents brief case reports that convey clear, practical lessons. Preference is given to common presentations of important rare conditions, and important unusual presentations of common problems. Articles start with a case presentation (500 words maximum), and a discussion of the underlying condition follows (1000 words maximum). Visual elements (e.g., tables of the differential diagnosis, clinical features or diagnostic approach) are encouraged. Consent from patients for publication of their story is a necessity. See information for authors at www.cmaj.ca. 